Proyecciones Journal of Mathematics

Vol. 35, No 3, pp. 245-249, September 2016.

Universidad Católica del Norte

Antofagasta - Chile

\title{
Unicyclic graphs with equal domination and complementary tree domination numbers
}

\author{
B. Krishnakumari \\ Sastra University, India \\ and \\ Y. B. Venkatakrishnan \\ Sastra University, India \\ Received : April 2015. Accepted : July 2016
}

\begin{abstract}
Let $G=(V, E)$ be a simple graph. A set $D \subseteq V(G)$ is a dominating set if every vertex in $V(G) \backslash D$ is adjacent to a vertex of $D$. A dominating set $D$ of a graph $G$ is a complementary tree dominating set if induced sub graph $\langle V \backslash D\rangle$ is a tree. The domination (complementary tree domination, respectively) number of $G$ is the minimum cardinality of a dominating (complementary tree dominating, respectively) set of $G$. We characterize all unicyclic graphs with equal domination and complementary tree domination numbers.
\end{abstract}

Keywords : Domination, complementary tree domination, unicyclic graphs.

AmS ; Subject Classification: 05C69. 


\section{Introduction}

Let $G=(V, E)$ be a graph. By the neighborhood of a vertex $v$ of $G$ we mean the set $N_{G}(v)=\{u \in V(G): u v \in E(G)\}$. The degree of a vertex $v$, denoted by $d_{G}(v)$, is the cardinality of its neighborhood. By a leaf we mean a vertex of degree one, while a support vertex is a vertex adjacent to a leaf. We denote $L(G)$ to be the set of leaves of the graph $G$ and $S(G)$ is the set of all support vertices of $G$. The path on $n$ vertices we denote by $P_{n}$. Let $T$ be a tree, and let $v$ be a vertex of $T$. We say that $v$ is adjacent to a path $P_{n}$ if there is a neighbor of $v$, say $x$, such that the subtree resulting from $T$ by removing the edge $v x$ and which contains the vertex $x$ as a leaf, is a path $P_{n}$.

A subset $D \subseteq V(G)$ is a dominating set of $G$ if every vertex of $V(G) \backslash D$ has a neighbor in $D$, while it is a complementary tree dominating set, abbreviated CTDS, of $G$ if the induced sub graph $\langle V \backslash D\rangle$ is a tree. The domination (complementary tree domination, respectively) number of a graph $G$, denoted by $\gamma(G)\left(\gamma_{c t d}(G)\right.$, respectively), is the minimum cardinality of a dominating (complementary tree dominating, respectively) set of $G$. A complementary tree dominating set of $G$ of minimum cardinality is called a $\gamma_{c t d}(G)$-set. The complementary tree domination in graphs was studied in [5]. For a comprehensive survey of domination in graphs, see $[1,2]$.

A unicyclic graph is a graph that contains exactly one cycle. In this paper we provide a constructive characterization of all unicyclic graphs with equal domination number and complementary tree domination number. In [3], unicyclic graphs with equal total and total outer-connected domination numbers are characterized.

\section{Preliminary results}

We begin with the following straightforward observation.

Observation 1. [5] Every leaf of a graph $G$ is in every $\gamma_{c t d}(G)$-set.

In [4] trees with equal domination number and complementary tree domination numbers are characterized. For this purpose the family $\mathcal{T}$ of trees $T=T_{k}$ is defined. Let $T_{1}$ be a path $P_{4}$. If $k$ is a positive integer, then $T_{k+1}$ can be obtained recursively from $T_{k}$ by one of the following operations. 
- Operation $\mathcal{O}_{1}$ : Attach a path $P_{2}$ by joining its any vertex to a vertex of $T_{k}$, which is not a leaf and is adjacent to a support vertex of degree two.

- Operation $\mathcal{O}_{2}$ : Attach a path $P_{2}$ by joining its any vertex to a support vertex of $T_{k}$.

Theorem 1. [4] Let $T$ be a tree. Then $\gamma_{c t d}(T)=\gamma(T)$ if and only if $T \in \mathcal{T}$.

\section{Unicyclic graphs}

We characterize all connected unicyclic graphs for which $\gamma(G)=\gamma_{c t d}(G)$. To this, we define $\mathcal{C}$ to be the family of all graphs $G$ for which exists a tree $T$ belonging to the family $\mathcal{T}$, such that $G$ is obtained from $T$ by the operation:

Operation $\mathcal{B}$ : Let $u, v$ be any two support vertices of $T$. Let $x$ and $y$ be the leaves adjacent to $u$ and $v$, respectively. Identify $x$ with $y$.

Let us also assume that $C_{3}$ and $C_{4}$ belong to $\mathcal{C}$ and observe that $C_{3}$ is obtained from $P_{4} \in \mathcal{T}$ by the above operation.

Lemma 2. If $G$ belong to the family $\mathcal{C}$, then $\gamma(G)=\gamma_{c t d}(G)$.

Proof. If $G$ is a cycle belonging to $\mathcal{C}$, then the result is immediate. Let us now assume that $G$ is obtained from a tree $T \in \mathcal{T}$ by Operation $\mathcal{B}$. Let $G$ be obtained from $T$ by identifying the leaves $x$ and $y$. Denote by $w$ the vertex obtained by identifying $x$ and $y$. It is easy to see that $L(G) \cup\{w\}$ is a minimum dominating set of $G$. Thus $\gamma(G)=|L(G)|+1$. On the other hand, $L(G) \cup\{w\}$ is a complementary tree dominating set of $G$. Thus we have $|L(G)|+1=\gamma(G) \leq \gamma_{c t d}(G) \leq|L(G)|+1$. Thus we have $\gamma(G)=\gamma_{c t d}(G)$.

Lemma 3. If $G$ is a connected unicyclic graph with $\gamma(G)=\gamma_{c t d}(G)$, then $G$ belongs to family $\mathcal{C}$.

Proof. Let $G$ be a connected unicyclic graph, where $C_{k}=\left(v_{1}, v_{2}, v_{3}, \cdots, v_{k}\right)$ is the unique cycle of $G$. Assume first that each vertex of $C_{k}$ is of degree 2. Then $G$ is a cycle $C_{k}$ for some $k \geq 3$. It is clear that $\gamma_{c t d}\left(C_{k}\right)=k-2$ for $k \geq 3$. On the other hand, $\gamma\left(C_{k}\right)<k-2$ for $k \geq 5$. Thus $\gamma\left(C_{k}\right)=\gamma_{c t d}\left(C_{k}\right)$ if $k \in\{3,4\}$. 
Assume that $G$ is not a cycle. If $v_{i} \in V\left(C_{k}\right)$, then let $T\left(v_{i}\right)$ be the tree obtained from $G$ by removing edges $v_{i} v_{i+1}$ and $v_{i-1} v_{i}$ (where the indices are taken modulo $k$ added 1 ) and containing $v_{i}$. Let $v_{i}$ be the root of $T\left(v_{i}\right)$. Let $D_{c t d}$ be a minimum complementary tree dominating set of $G$.

Assume without loss of generality, that $d_{G}\left(v_{1}\right) \geq 3$, and denote by $x$ any element of $V\left(T\left(v_{1}\right)\right)$ which is neither a leaf nor a support vertex. Let $x \in D_{c t d}$. Then either $V(G) \backslash D_{c t d} \subseteq V(T(x))$ or $V(G) \backslash D_{c t d} \subseteq$ $V(G) \backslash V(T(x))$, Let $V(G) \backslash D_{c t d} \subseteq V(G) \backslash V(T(x))$. It is clear that $V(G) \backslash D_{c t d}$ contains a cycle, contradiction to the definition of $D_{c t d}$. Now assume $V(G) \backslash D_{c t d} \subseteq V(T(x))$. Then $D_{c t d} \backslash\{u\}$ where $u$ is a leaf in $T(x)$ is a dominating set of $G$ of smaller cardinality than $\gamma(G)$, a contradiction. Let $x \notin D_{c t d}$. Arguing as above, we get a contradiction. Hence, we conclude that every vertex in $T\left(v_{1}\right)$ is either a support vertex or a leaf.

Assume $V(C) \cap D_{c t d}=\phi$. The complement of $D_{c t d}$ contains a cycle, a contradiction. Now assume, without loss of generality, that $v_{1} \in V(C) \cap$ $D_{c t d}$ and $d_{G}\left(v_{1}\right) \geq 3$. Then obviously $V\left(T\left(v_{1}\right)\right) \subseteq D_{c t d}$. It is easy to see that $D_{c t d} \backslash\{u\}$ where $u$ is a leaf in $T\left(v_{1}\right)$ is a dominating set of $G$ of smaller cardinality than $\gamma(G)$, a contradiction. Hence, $d_{G}\left(v_{1}\right)=2$.

Now assume $d_{G}\left(v_{2}\right) \geq 3$ and $d_{G}\left(v_{k}\right) \geq 3$. Suppose $v_{2}$ and $v_{k}$ is in $D_{c t d}$, then $D_{c t d} \backslash\left\{v_{1}\right\}$ is a dominating set of cardinality smaller than $\gamma(G)$, a contradiction. Without loss of generality, assume $v_{2} \in D_{\text {ctd }}$. Arguing as in the previous case, we get $d_{G}\left(v_{2}\right)=2$. Assume that $v_{3}$ and $v_{k}$ not in $D_{c t d}$. Then since $D_{c t d}$ is a complementary tree dominating set, exactly two vertices of $V\left(C_{k}\right)$ belong to $D_{c t d}$, namely $v_{1}$ and $v_{2}$. It is easy to see that $T\left(v_{i}\right) \backslash\left\{v_{i}\right\} \in D_{c t d}, 3 \leq i \leq k$. It is obvious that $D_{c t d} \backslash\left\{v_{2}\right\}$ is a dominating set of cardinality smaller than $\gamma(G)$, a contradiction. Thus $v_{1}$ is the only vertex in $D_{c t d}$ set of $G$.

Denote by $G_{1}$ the graph obtained from $G$ by removing the edge $v_{1} v_{2}$ and attaching the vertex $x$ to the vertex $v_{2}$. It is obvious that $\gamma(G) \leq$ $\gamma\left(G_{1}\right)$. Suppose $D_{c t d}$ is a $\gamma_{c t d}\left(G_{1}\right)$-set of cardinality smaller than $\gamma_{c t d}(G)+1$. The vertex $x$ is a leaf in $G_{1}$. By observation 1 , the leaf $x \in D_{c t d}$. Then $D_{c t d}^{\prime}=D_{c t d} \backslash\{x\}$ is a complementary tree dominating set of $G$. Thus $\gamma(G)=\gamma_{c t d}(G) \leq\left|D_{c t d}^{\prime}\right| \leq \gamma_{c t d}\left(G_{1}\right)-1$. It is easy to observe that $D_{c t d}^{\prime} \cup\{x\}$ is a complementary tree dominating set of $G_{1}$, so $\gamma_{c t d}\left(G_{1}\right) \leq \gamma_{c t d}(G)+1$. Equivalently, $\gamma_{c t d}(G) \geq \gamma_{c t d}\left(G_{1}\right)-1$. This implies that $\gamma_{c t d}(G)=\gamma_{c t d}\left(G_{1}\right)-$ 1. Since $G_{1}$ is a tree, theorem 1 implies that $G_{1}$ belongs to the family $\mathcal{T}$. We conclude that $G$ is obtained from a tree belonging to the family $\mathcal{T}$ by operation $\mathcal{B}$. Therefore, $G$ belongs to the family $\mathcal{C}$. 


\section{References}

[1] T. Haynes, S. Hedetniemi and P. Slater, Fundamentals of Domination in Graphs, Marcel Dekker, New York, (1998).

[2] T. Haynes, S. Hedetniemi and P. Slater (eds.), Domination in Graphs: Advanced Topics, Marcel Dekker, New York, (1998).

[3] Joanna Raczek, Unicyclic graphs with equal total and total outerconnected domination numbers, Ars Comb 118, pp. 167-178, (2015).

[4] B. Krishnakumari and Y.B. Venkatakrishnan, A note on complementary tree domination number of tree, Proyecciones Journal of Mathematics 34 (2), pp. 127-136, (2015).

[5] S. Muthammai, M. Bhanumathi and P. Vidhya, Complementary tree domination number of a graph, International Mathematical Forum 6, pp. 1273-1282, (2011).

\section{B. Krishnakumari}

Department of Mathematics, School of Humanities and Sciences, SASTRA University, Thanjavur-613 401, Tamilnadu, India

e-mail : krishnakumari@maths.sastra.edu

and

\section{Y. B. Venkatakrishnan}

Department of Mathematics, School of Humanities and Sciences, SASTRA University, Thanjavur-613 401, Tamilnadu, India

e-mail : ybvenkatakrishnan2@gmail.com 